\title{
Influence of Surface Pretreatment on Residual Stress Field of Heat-Treated Steel Induced by Laser Local Quenching
}

\author{
Yoshihisa Sakaida ${ }^{1, a,{ }^{*}}$, Yuki Sasaki ${ }^{2, b}$ and Haruo Owashi ${ }^{3, c}$ \\ ${ }^{1}$ Dept. of Mech. Eng., Shizuoka University, Naka-ku, Hamamatsu 432-8561, Japan \\ ${ }^{2}$ Grad School of Eng., Shizuoka University, Japan \\ ${ }^{3}$ Atsumi Kogyo Co. Ltd., Higashi-ku Hamamatsu 435-0042, Japan \\ a sakaida.yoshihisa@shizuoka.ac.jp, b yuki.sasaki.16@gmail.com, ${ }^{\text {c }}$ owashi@atsumi-kogyo.co.jp
}

Keywords: Laser Quenching, Carbon Steel, Residual Stress Field, Surface Pretreatment, X-Ray Stress Measurement, Quenching Temperature

Abstract. In order to clarify an influence of the surface pretreatment condition on the residual stress field after laser local quenching, three kinds of surface pretreatment were applied to the steel specimens. In this study, the mirror-polished, matte-black-painted and mechanical-polished surfaces were prepared, and were heat-treated by two times laser irradiation. After quenching, the irradiation marks and hardened layers were observed. The irradiation mark on the mirrorpolished surface was insufficient. On the other hand, the hardened layers under the matte-black painted and mechanical-polished surfaces were formed enough. The residual stress fields of the latter two specimens were measured. As a result, surface pretreatment condition was found to make a big difference in both the $2 \theta-\sin ^{2} \psi$ diagram and the residual stress field after laser quenching. Therefore, in order to investigate these causes, surface temperature histories during laser irradiation were monitored using a radiation thermometer. From these results, it was clarified that the difference of the first heat-treatment temperature directly affects the presence or absence of residual stress gradient and the depth of hardened layer under the laser-irradiated surface. Finally, adequate surfaces for the steel parts before laser local quenching could be proposed.

\section{Introduction}

Laser local quenching [1] is very useful technique to improve the surface strengths of the local area, such as hardness, fatigue and fracture strengths, of steel parts having a complicated shape. The strengthening effect due to laser quenching depends directly on the hardness and compressive residual stress distributions near the quenched surface. However an influence that laser and surface pretreatment conditions give to intensities of hardness and compressive residual stress field is not sufficiently clear. In this study, the surface pretreatment condition is thought to give a maximum influence on the improved surface strength after quenching. Three kinds of pretreatment having different heat-absorptivity were applied to the surface of the steel specimens. These pretreated specimens were heat-treated by two times laser irradiation. After second laser irradiation, the residual stress field of each specimen was measured experimentally using x-ray stress measurement. An influence of surface pretreatment condition on the residual stress field and the hardness distribution under the quenched surface were examined.

\section{Experimental Procedure}

Materials and Specimens. The initial material was a carbon steel with 0.45 mass\% C, 0.21 mass $\% \mathrm{Si}$, and 0.81 mass $\% \mathrm{Mn}$. At first, the material was normalized twice from $900^{\circ} \mathrm{C}$ in air, and was quenched from $870^{\circ} \mathrm{C}$ in oil at $80^{\circ} \mathrm{C}$. Before laser quenching, the material was changed from martensite to fine pearlite by tempering from $550^{\circ} \mathrm{C}$ in air. The plate specimens with a 
width of $25 \mathrm{~mm}$ and a thickness of $10 \mathrm{~mm}$ was used. In this study, three kinds of different surfaces were prepared. One is mirror-polished surface. Another is matte-black-painted surface with soluble acrylic resin. The other is mechanical-polished surface with \#600 sandpaper.

Laser Local Quenching. These specimens were heat-treated in air by two times laser irradiation as shown in Fig. 1. Each laser irradiation was conducted at a laser power, $P_{L}=1 \mathrm{~kW}$, on the local area of the pretreated surface using a semiconductor laser processing machine with a direct diode laser (L1, Enshu Ltd., Japan). In experiment, a rectangular laser beam about $\mathrm{W}_{\mathrm{L}}=3.9 \mathrm{~mm}$ and $\mathrm{W}_{\mathrm{T}}=8.8 \mathrm{~mm}$ was used. An incident angle, $\delta$, and a scanning speed, $V_{F}$, were 15 or 45 degrees and $1.0 \mathrm{~m} / \mathrm{min}$, respectively. By scanning the laser beam, the local area was heated and quenched immediately by self-cooling. After first laser irradiation, the laser beam was returned to the starting point. The local area was heated and quenched again by second laser irradiation.

X-ray Stress Measurement. After second laser irradiation, the $2 \theta-\sin ^{2} \psi$ diagrams were measured experimentally from the irradiation mark using an X-ray stress measurement equipment with $\Omega$ - goniometer (MSF-2M-PSPC, Rigaku Co. Ltd., Japan). Residual stress field of each specimen was estimated from the $2 \theta-\sin ^{2} \psi$ diagrams at several $\varphi$-angles, as shown in Fig. 2. Table 1 summarizes $\mathrm{x}$-ray stress measurement condition. In this study, the diffraction from 211 plane of martensite by $\mathrm{Cr}-\mathrm{K} \alpha$ radiation was used. The x-ray Young's modulus, $E_{X}$, and Poisson's ratio, $v_{X}$, of 211 plane of martensite were $186 \mathrm{GPa}$ and 0.30 , respectively [2]. The existences of $\psi$ splitting phenomenon and stress gradients near the quenched surface were examined from the $2 \theta$ $\sin ^{2} \psi$ diagrams.

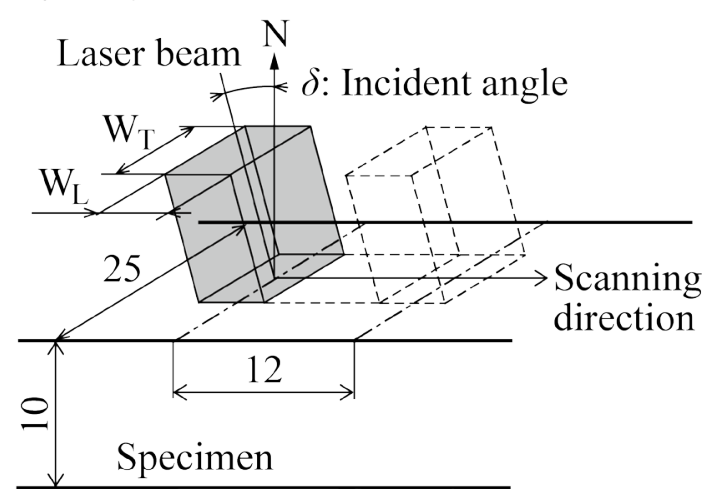

Table $1 X$-ray stress measurement condition for laser local quenching.

\begin{tabular}{l|l}
\hline Characteristic x-ray & $\mathrm{Cr}-\mathrm{K} \boldsymbol{\alpha}$ \\
Tube voltage $(\mathrm{kV})$ & 30 \\
Tube current $(\mathrm{mA})$ & 8 \\
Diffraction & $\mathrm{Fe}-211$ \\
Diffraction angle $(\mathrm{deg})$ & 155.57 \\
Range of $\sin ^{2} \boldsymbol{\psi}$ & $0-0.75$ \\
Step in $\sin ^{2} \boldsymbol{\psi}$ & 0.05 \\
$\boldsymbol{\varphi}$ angle (rad) & $0, \pi / 4, \pi / 2,3 \pi / 4, \pi$ \\
& $5 \pi / 4,3 \pi / 2,7 \pi / 4$ \\
Incident collimator $\left(\mathrm{mm}^{2}\right)$ & $2 \times 2$ \\
\hline
\end{tabular}

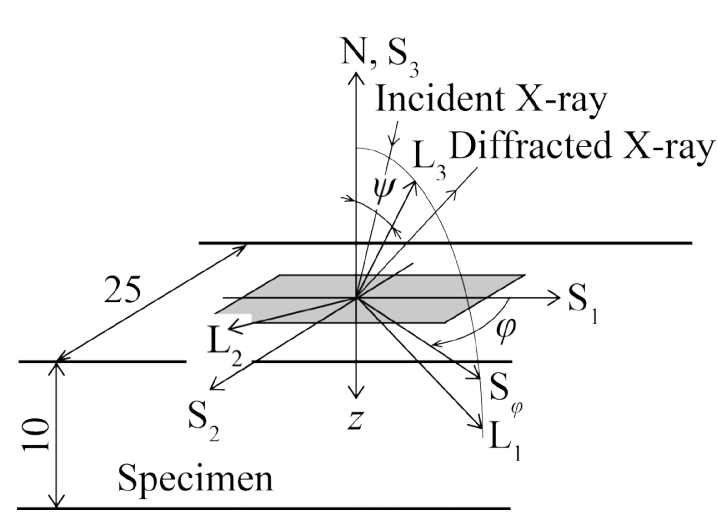

Fig.1 Local area heating and quenching by

Fig.2 Laboratory and sample coordinate scanning a semiconductor laser beam. systems, $L_{i}$ and $S_{i}$, and $\psi$ and $\varphi$-angles.

\section{Results and Discussion}

\section{Irradiation Mark of Laser Beam and Hardened Layer Formed near the Surface.}

Figures 3(a), 3(b) and 3(c) show images of the irradiation mark of the laser beam formed on the different surfaces. On the mirror-polished surface, the irradiation mark at $\delta=45 \mathrm{deg}$ resembles a teardrop shape. The hardened layer below the teardrop area was insufficient because the incident laser beam scattered on the mirror surface and the heat-treatment temperature near the surface did not rise to austenite phase. On the other hand, other irradiation mark shapes are rectangular, and are larger than that of the mirror polished surface at $\delta=45 \mathrm{deg}$. Therefore, matteblack painting and mechanical polishing were more adequate pretreatments than mirror 
polishing, because the laser quenched mark was stable even though the incidence angle of laser beam changed. Next, using a wire-electrical discharge machine, the matte-black-painted and mechanical-polished specimens after second laser irradiation were cut to observe the hardened layer.

Figures 4(a) and 4(b) show the cross-sectional micrographs of two different pretreated specimens. At first, the hardened layer of the matte-black-painted specimen was found to be deeper than that of the mechanical-polished specimen. Furthermore, the depth of hardened layer at $\delta=15 \mathrm{deg}$ was found to be deeper than that at $\delta=45 \mathrm{deg}$. Therefore, two kinds of pretreated specimen quenched at $\delta=15 \mathrm{deg}$ were picked up. X-ray stress measurements were carried out on the two quenched marks at the next step.

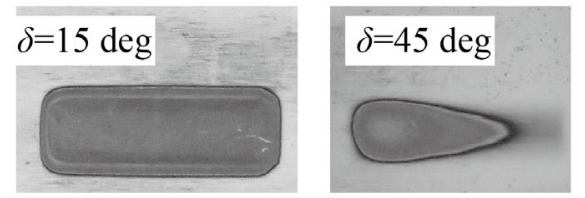

(a) On the mirror-polished surface

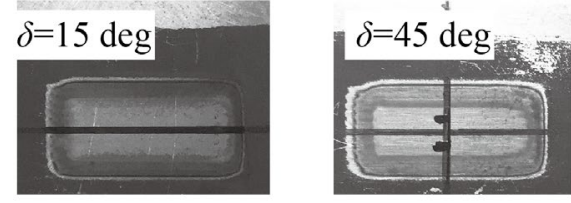

(b) On the matte-black-painted surface

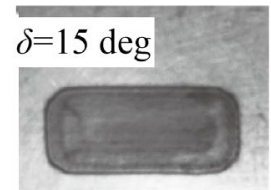

(c) On the mechanical-polished surface

Fig.3 Photographs of irradiation mark of laser beam formed on the surfaces.

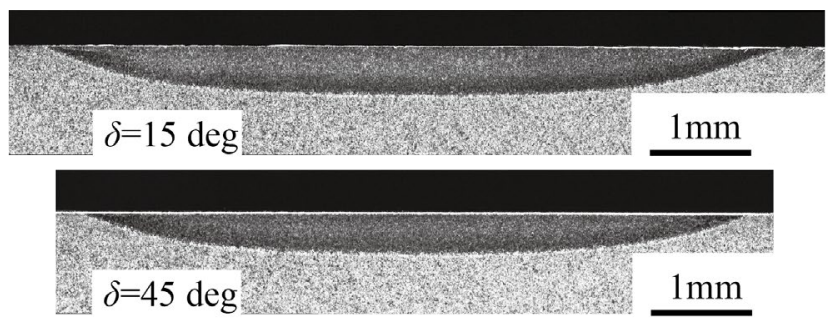

(a) Near the matte-black-painted surface

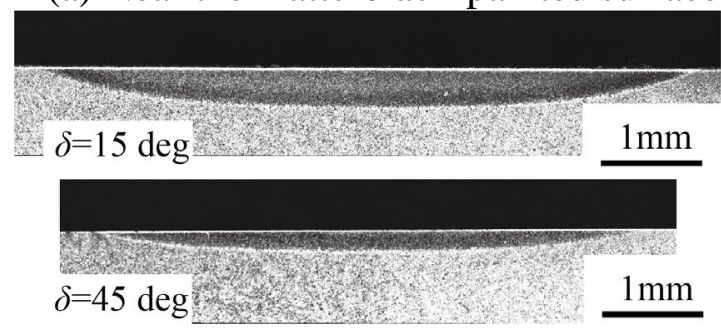

(b) Near the mechanical-polished surface

Fig.4 Cross-sectional micrographs of hardened layer below the irradiation marks.

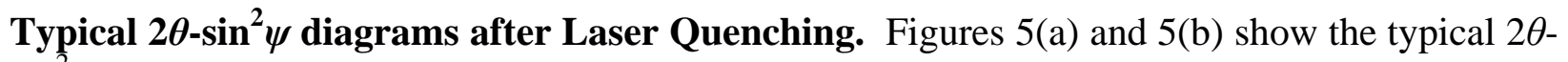
$\sin ^{2} \psi$ diagrams at $\delta=15 \mathrm{deg}$ from the matte-black-painted and mechanical-polished specimens, respectively. In each figure, the first, second, third and fourth figures from the left show the results for $\pm \psi$ at $\varphi=0, \pi / 4, \pi / 2$ and $3 \pi / 4$, respectively. These four pair figures were used to judge the existences of $\psi$-splitting phenomenon and stress gradient.

In the case of the matte-black-painted specimen as shown in Fig. 5(a), each pair figure almost overlaps, and the overlapped $2 \theta-\sin ^{2} \psi$ diagram exhibits a convex upward quadratic function. On the other hand, in the case of the mechanical-polished specimen as shown in Fig. 5(b), each pair figure almost overlaps too, but the overlapped $2 \theta-\sin ^{2} \psi$ diagram exhibits a linear function. Therefore, the $\psi$-splitting could not be observed in all $2 \theta-\sin ^{2} \psi$ diagrams. After two times laser quenching, the matte-black- painted specimen was found to have a stress gradient below the quenched mark, while mechanical- polished specimen did not have a stress gradient.

Estimation of Residual Stress Fields after Laser Quenching. The residual stress field near the quenched mark of the matte-black-painted specimen was first estimated from four pair figures 5(a). In this case, the biaxial principal compressive residual stresses were thought to be generated near the quenched surface and to have constant stress gradients because these $2 \theta-\sin ^{2} \psi$ diagrams could be approximated as quadratic functions. In this study, three residual stress components, normal stresses, $\sigma_{11}(\mathrm{z}), \sigma_{22}(\mathrm{z})$ and shear stress, $\sigma_{12}(\mathrm{z})$, near the local quenched surface were defined as, 

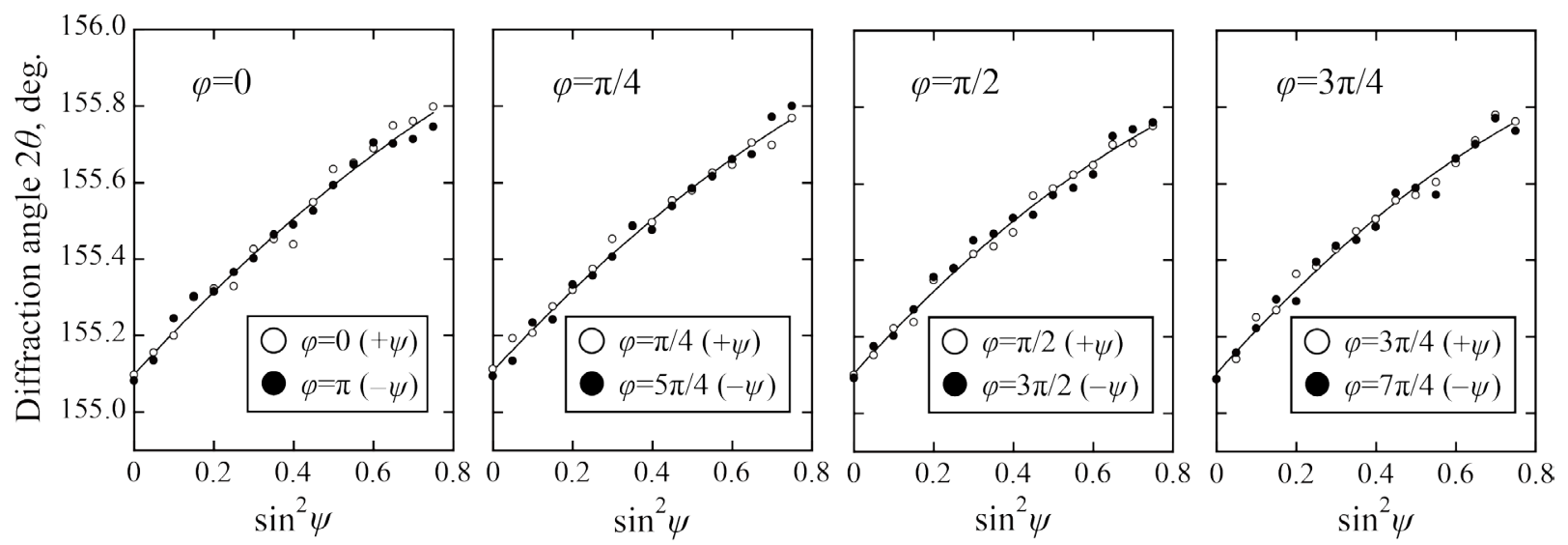

(a) On the matte-black-painted specimen.
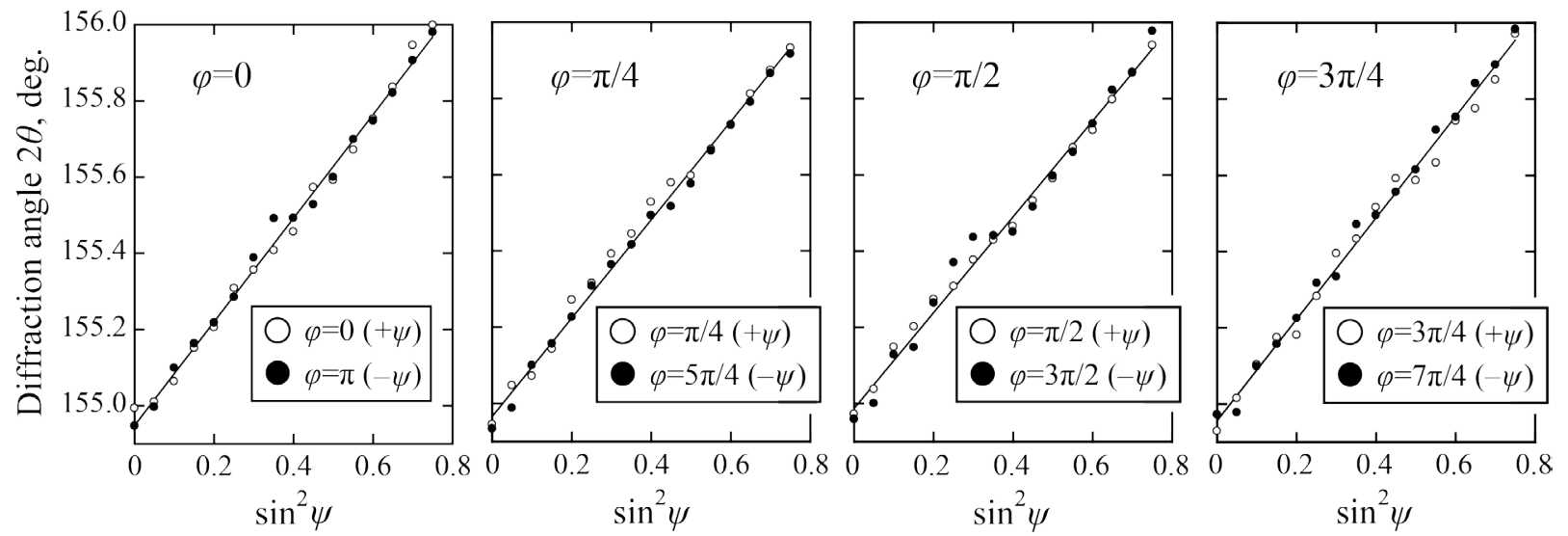

(b) On the mechanical-polished specimen.

Fig. 5 The $2 \theta-\sin ^{2} \psi$ diagrams from the quenched marks by laser beam at $\delta=15 \mathrm{deg}$.

$$
\sigma_{i j}(z)=\left(\begin{array}{ll}
\sigma_{11}(0) & \sigma_{12}(0) \\
\sigma_{12}(0) & \sigma_{22}(0)
\end{array}\right)+\left(\begin{array}{ll}
A_{11} & A_{12} \\
A_{12} & A_{22}
\end{array}\right) z,
$$

where $\sigma_{i j}(0)$ is residual stress component on the surface and $A_{i j}$ is a constant stress gradient. In the case of stress field having stress gradients, the measured stress parallel to the $S_{\varphi}$-direction is the weighted average stress $\left\langle\sigma_{i j}>\right.$, as follows [3, 4],

$$
<\sigma_{i j}>=\left[\int_{0}^{t} \sigma_{i j}(z) \cdot \exp \left(-\frac{z}{T_{0}}\right) d z\right] /\left[\int_{0}^{t} \exp \left(-\frac{z}{T_{0}}\right) d z\right]=\sigma_{i j}(0)+A_{i j} T_{0} W,
$$

where $W$ is weighted coefficient. $W$ is equal to 1.0 at $t=\infty[4]$. $T_{0}$ is the effective x-ray penetration depth with $\Omega$-goniometer is expressed as [3, 4],

$$
T_{0}=\left(\sin ^{2} \theta_{0}-\sin ^{2} \psi\right) /\left(2 \mu \sin \theta_{0} \cos \psi\right)=T_{0}+T_{1} \sin ^{2} \psi,
$$

where $\mu$ is a linear absorption coefficient, $2 \theta_{0}$ is stress-free diffraction angle estimated from the measured $2 \theta-\sin ^{2} \psi$ diagrams, $T_{1}$ and $T_{0}$ are regression coefficient and intercept that are determined by a least square method. In this case, $\mu=844 \mathrm{~cm}^{-1}, 2 \theta_{0}=155.57$ deg., $T_{0}=5.95 \mu \mathrm{m}$ and $T_{1}=-4.30 \mu \mathrm{m}$, respectively.

Next, four overlapped $2 \theta$-sin $2 \psi$ diagrams for $\varphi=0, \pi / 4, \pi / 2$ and $3 \pi / 4$ were fitted as quadratic functions by a least square method. Three residual stress components on the surface and constant stress gradients were determined experimentally $[3,4]$. As a result, the residual stress field was obtained as follows, 


$$
\left.\sigma_{i j}(z)=\left(\begin{array}{cc}
-151.9 & -6.8 \\
-6.8 & -124.1
\end{array}\right)+\left(\begin{array}{cc}
-19.1 & 2.5 \\
2.5 & -24.1
\end{array}\right) z \text {, [units of } \sigma_{i j}(0): \mathrm{MPa}, A_{i j}: \mathrm{MPa} / \mu \mathrm{m}\right] .
$$

The compressive residual stresses about $\sigma_{11}(0)=-152 \mathrm{MPa}$ and $\sigma_{22}(0)=-124 \mathrm{MPa}$ were generated parallel to the $S_{1}$ and $S_{2}$ directions on the matte-black-painted surface. The residual stress gradients abut $A_{11}=-19 \mathrm{MPa} / \mu \mathrm{m}$ and $A_{22}=-24 \mathrm{MPa} / \mu \mathrm{m}$ were also generated near the quenched surface. The compression residual normal stress components were found to increase with an increase in the depth from the local quenched surface.

On the other hand, the residual stress field near the quenched surface of the mechanicalpolished specimen was estimated from four pair figures 5(b). In that case, the constant biaxial principal compressive residual stresses were thought to be generated from the local quenched surface to the effective $x$-ray penetration depth because those $2 \theta-\sin ^{2} \psi$ diagrams could be approximated as linear functions. Therefore, four overlapped $2 \theta-\sin ^{2} \psi$ diagrams for $\varphi=0, \pi / 4, \pi / 2$ and $3 \pi / 4$ were fitted as linear functions by a least square method. Three residual stress components were determined experimentally. As a result, the residual stress field was obtained as follows,

$$
\sigma_{i j}=\left(\begin{array}{cc}
-367.3 & 5.7 \\
5.7 & -339.6
\end{array}\right) \text {, [unit of } \sigma_{i j}: \text { MPa]. }
$$

The large compressive residual stresses about $\sigma_{11}=-367 \mathrm{MPa}$ and $\sigma_{22}=-340 \mathrm{MPa}$ were generated parallel to the $S_{1}$ and $S_{2}$ directions on the mechanical-polished surface.

Heat-treatment Temperature during Irradiating Laser Beam. Surface pretreatment condition before laser quenching was found to make a big difference in both the $2 \theta-\sin ^{2} \psi$ diagram and the residual stress field after two laser quenching. Therefore, surface temperatures, $T_{1}$ and $T_{2}$, at the center were monitored immediately after two times laser irradiation using a radiation thermometer with an emissivity of 0.8 . Figure 6 shows the comparison of apparent temperature changes of two kinds of pretreated specimens. It was found that $T_{1}$ of the matteblack -painted specimen was much higher than that of the mechanical-polished specimen. It is thought that difference of $T_{1}$ results from high heat-absorptivity of the matte-black-painted surface. On the other hand, $T_{2}$ of two specimens was about $1070^{\circ} \mathrm{C}$. The mat-black coating on the

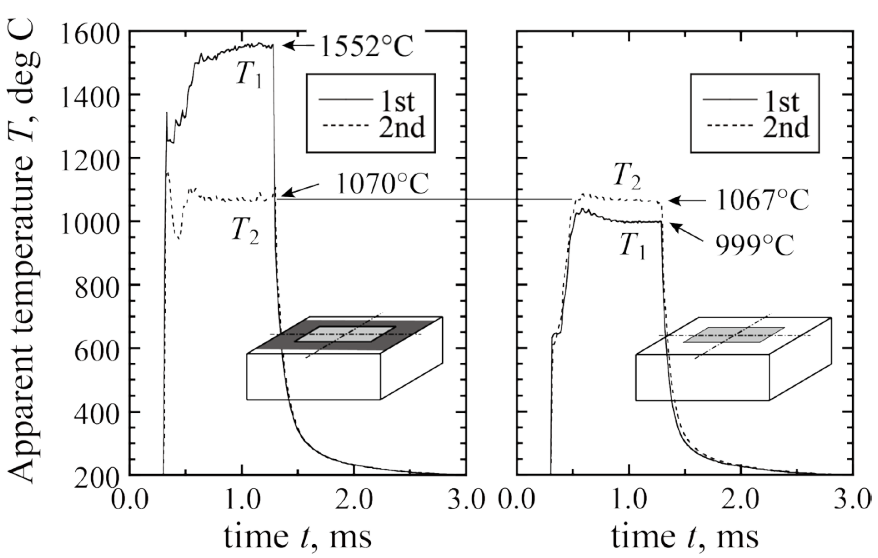

Fig.6 Comparison of surface temperatures, $T_{1}$ and $\mathrm{T}_{2}$, after 1st and 2nd laser irradiation at $\delta=15 \mathrm{deg}$.

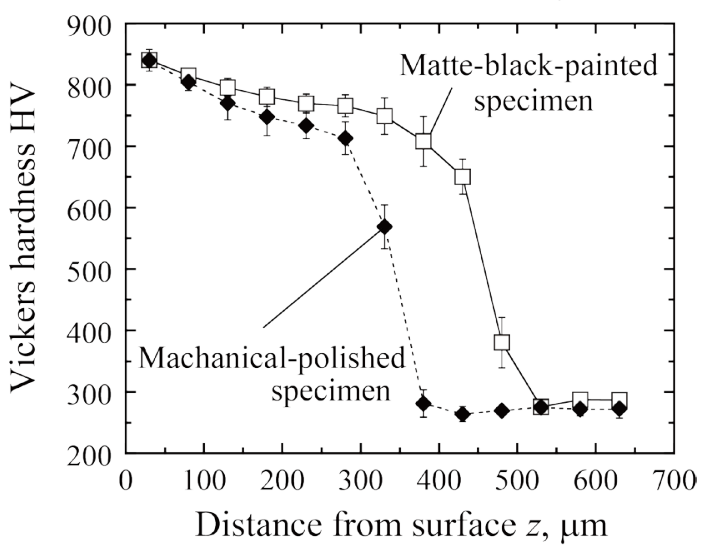

Fig.7 Vickers hardness distributions of hardened layers in two specimens.

surface was sublimated during first laser irradiation, so that $T_{2}$ dropped to $1070^{\circ} \mathrm{C}$ due to the reduced heat-absorptivity of the first quenched mark. In contrast, the first quenched mark also put on the surface of the mechanical- polished specimen, and then $T_{2}$ slightly rose to $1067^{\circ} \mathrm{C}$ due 
to the enlarged heat-absorptivity.

Figure 7 shows Vickers hardness distributions of hardened layers in both matte-black-painted and mechanical-polished specimens. The surface hardness of two specimens was equal to $840 \mathrm{HV}$ because of same $T_{2}$ before second self-cooling. However, both first-heating temperature $T_{1}$ and total amount of heat, $Q$, absorbed on the matte-black painted surface were higher and larger than $T_{1}$ and $Q$ on the mechanical-polished surface. Higher heat-treatment temperature, $T_{1}$, and larger amount of absorbed heat were found to give rise to compressive residual stress field having a constant stress gradient and the deeper hardened layer near the matte-black-painted surface. Therefore, it is concluded that matte-black painting is more adequate pretreatment from the viewpoint that the depth of hardened layer is preferably deeper. In contrast, it is also concluded that mechanical polishing is more adequate pretreatment from the viewpoint that the compressive residual stress on the surface is preferably higher for the surface strength of mechanical parts.

\section{Conclusions}

Three kinds of pretreated specimens having different surfaces were heat-treated by two times laser irradiation. The residual stress field near the irradiation mark and hardness distribution were measured experimentally. The results are summarized as follows:

(1) Matte-black painting and mechanical polishing were more adequate surface-pretreatments than mirror polishing, because the irradiation marks on the matte-black-painted and mechanicalpolished surfaces were stable even though the incidence angle of laser beam changed.

(2) Surface pretreatment condition was found to make a big difference in the $2 \theta-\sin ^{2} \psi$ diagram and the residual stress field after laser quenching. The $2 \theta-\sin ^{2} \psi$ diagram of the matte-blackpainted specimen exhibits a quadratic function. The biaxial compressive residual stress field having a constant stress gradient was found to be generated. On the other hand, the $2 \theta$ - $\sin ^{2} \psi$ diagram of mechanical-polished specimen exhibits a linear function. The constant biaxial compressive residual stress field was found to be generated within the effective x-ray penetration depth.

(3) By measuring the heat-treatment temperature, higher temperature and larger amount of absorbed heat were found to give rise to compressive residual stress field having a stress gradient and the deeper hardened layer near the matte-black painted surface. Therefore, matte-black painting is found to be more adequate pretreatment from the viewpoint that the depth of hardened layer is preferably deeper. In contrast, mechanical polishing is found to be more adequate pretreatment from the viewpoint that the compressive residual stress on the surface is preferably higher.

\section{References}

[1] Edited by the Japan Society of Mech. Eng., Thermomechanical behavior of materials during transformation, theory and numerical simulation, Corona Pubishing. Co., Ltd: Tokyo, (1991), 142-162.

[2] Y. Sakaida and K. Tanaka, Change of x-ray elastic constants of carbon steel caused by quenching and effect of its change on X-ray stress measurement, J. the Society of Materials Science, Japan, 56-7 (2007) 602-608. https://doi.org/10.2472/jsms.56.602

[3] I. C. Noyan and J. B. Cohen, Residual stress; measurement by diffraction and interpretation, Springer-Verlag: New York, (1987), p. 134. https://doi.org/10.1007/978-1-4613-9570-6

[4] K. Suzuki, K. Tanaka and Y. Sakaida, Penetration depth in X-ray analysis of steep stress gradient near surface, J. the Society of Materials Science, Japan, $45-7$ (1996) 759-765. https://doi.org/10.2472/jsms.45.759 